Bull. Mater. Sci., Vol. 36, No. 4, August 2013, pp. 559-562. (C) Indian Academy of Sciences.

\title{
Low-field magnetization process and complex permeability of FeCoBSiTa wires coated with hard magnetic CoNi layer
}

\author{
S BÁEZ-PIMIENTO*, I BETANCOURT, M E HERNÁNDEZ-ROJAS ${ }^{\dagger}$, \\ G A BADINI-CONFALONIERI ${ }^{\#}$, A JACAS ${ }^{\#}$ and M VÁZQUEZ\# \\ Departamento de Materiales Metálicos y Cerámicos, Instituto de Investigaciones en Materiales, Universidad Nacional \\ Autónoma de México, México D.F. 04510, México \\ ${ }^{\dagger}$ Departamento de Recursos de la Tierra, Universidad Autónoma Metropolitana - Lerma, Estado de México 52006, México \\ \#Instituto de Ciencia de Materiales de Madrid, CSIC, 28049 Madrid, Spain
}

MS received 16 December 2011; revised 15 June 2012

\begin{abstract}
Biphase wires consisting of a soft magnetic amorphous nucleus surrounded by a hard magnetic CoNi layer of variable thickness were obtained by means of rotating water-quenching method and subsequent electroplating technique. Magnetization processes for all the biphase wires were resolved in terms of reversible bulging of magnetic domains and spin rotation by means of complex permeability measurements within the frequency range of $10 \mathrm{~Hz}-13 \mathrm{MHz}$. Results are interpreted in terms of CoNi layer effect on the magnetic anisotropy of the soft core.
\end{abstract}

Keywords. Biphase wires; soft/hard magnetic materials; amorphous wires.

\section{Introduction}

Amorphous metallic materials hold a prominent position among functional alloys because of their favourable combination of mechanical, electrical, magnetic and chemical properties, derived from the absence of atomic long range order (Konno et al 1990; Hernando and Marín 2005; Pirota et al 2005; Vazquez 2007). In particular, Fe-based amorphous alloys in the shape of wires with diameters of around $0.1 \mathrm{~mm}$, obtained by means of rotating water-quenching process, present an outstanding soft magnetic behaviour including high magnetization saturation and large magnetic permeability values besides very low coercivity. In addition, these ultra-soft magnetic amorphous wires possess a characteristic magnetic domain structure consisting of a central core with a longitudinal domain structure surrounded by a sheath of perpendicular magnetization with either radial or circular orientation depending on the sign of the wire's saturation magnetostriction, $\lambda_{\mathrm{s}}\left(\lambda_{\mathrm{s}}>0\right.$ for radial direction and $\lambda_{\mathrm{s}}<0$ for circular orientation, whereas vanishing $\lambda_{\mathrm{s}}$ destroys the single inner domain structure) (Konno et al 1990; Hernando and Marín 2005; Pirota et al 2005; Vazquez 2007). These magnetic features have led to the implementation of a variety of technological applications for these materials as active elements in magnetic sensors and/or transducer devices.

Recently, amorphous wires have elicited a considerable interest in the development of biphase wires consisting of a soft magnetic metallic nucleus (Fe-based amorphous wire) surrounded by a hard magnetic layer deposited by electrochemical process (Pirota et al 2004). This hard magnetic

\footnotetext{
*Author for correspondence (sbp@iim.unam.mx)
}

layer allows the control of the biphase wires magnetic properties by its magnetic interaction with the soft magnetic nucleus through: (i) the modification of the easy axis of magnetization of the wire from radial to longitudinal orientation depending on the hard layer thickness (i.e. on the electrochemical deposition parameters, viz. current density and time of electrochemical exposure), (ii) the possibility of obtaining controllable magnetostatic or eventually exchange bias response (similar to the bias effect used in spin valves) and (iii) the tailoring of their magnetic domain formation and thus, their magnetic performance from non-hysteretic to bistable behaviour (Vázquez et al 2001).

In earlier studies, attention was focused on biphase wire systems where the core phase is prepared by quenching and drawing technique containing an insulating interphase layer. However, less attention was paid to alternative biphase systems where the core is prepared by the in-rotating-water quenching technique where both soft and hard phases are in direct contact (Vázquez et al 2007). This paper presents a detailed study on the low-field magnetization process of a particular biphase soft/hard system for which external magnetic layer is directly made to grow on the amorphous core. This certainly corresponds to the behaviour of the soft core under the influence of the harder shell. Particularly, we pay attention to the low-field hysteresis loop and complex permeability.

\section{Sample preparation and experimental techniques}

Amorphous wires with nominal composition, $\mathrm{Fe}_{64} \mathrm{Co}_{8} \mathrm{~B}_{19.2} \mathrm{Si}_{4.8} \mathrm{Ta}_{4}$ and $140 \mu \mathrm{m}$ in diameter, were obtained 
in our laboratories by the in-rotating water quenching method at $2600 \mathrm{rpm}$ circular velocity under 4.25 Psi injection pressure. The as-cast wires were subsequently subjected to an electroplating process to deposit a $\mathrm{Co}_{90} \mathrm{Ni}_{10}$ magnetic hard layer using a chemical bath composed of $\mathrm{NiCl}_{2}, \mathrm{NiSO}_{4}$, $\mathrm{H}_{3} \mathrm{BO}_{3}, \mathrm{CoCl}_{2}$ and $\mathrm{CoSO}_{4}$ under an electric current density of $12 \mathrm{~mA} / \mathrm{cm}^{2}$ with exposure times of 5,10 and $30 \mathrm{~min}$, for which layers of thickness, $t=1 \cdot 5,3$ and $6 \mu \mathrm{m}$, respectively were generated. Earlier studies by energy dispersive spectroscopy (EDS) showed that the deposited CoNi layers correspond to crystalline phase (Torrejón 2008). On the other hand, in the same research, composition analysis of the generated CoNi layer, performed by EDS, indicated a content of $10 \% \mathrm{Ni}$ when the electric current density was constant $\left(j=12 \mathrm{~mA} / \mathrm{cm}^{2}\right)$ to exposure time at a range of 0.0-60 min (Torrejón 2008). The soft nucleus diameter and hard CoNi layer thickness were measured by scanning electron microscopy (SEM) (Jeol JSM 7600F) operated at $20 \mathrm{keV}$ and $700 \mathrm{pA}$. The magnetic properties (i.e. $M-H$ hysteresis loops and their parameters) were measured by inductive methods at $50 \mathrm{~Hz}$ using $M-H$ loop tracer based on digital signal processing for low frequency, which reaches a maximum magnetic field of $2.1 \mathrm{kA} / \mathrm{m}$ (Butta et al 2009). Complex permeability, $\mu^{*}=\mu_{\mathrm{re}}+j \mu_{\mathrm{im}}$, was determined from complex impedance, $Z^{*}=Z_{\mathrm{re}}+j Z_{\mathrm{im}}$, measured according to the relation (Valenzuela 2004, 2007):

$$
\mu^{*}=G\left(\frac{-j Z^{*}}{\omega}\right)
$$

where $G$ is an appropriate geometrical factor and $\omega$ denotes the angular frequency. Impedance measurements were carried out with a HP4192A impedance analyser at a frequency range of $10 \mathrm{~Hz}-13 \mathrm{MHz}$ under an a.c. applied field, $H_{\mathrm{ac}}=$ $0.5 \mathrm{~A} / \mathrm{m}$, along the wire's longitudinal direction.

\section{Results and discussion}

Figure 1 shows soft nucleus with $\sim 140 \mu \mathrm{m}$ in diameter and the external hard CoNi layer with $3 \mu \mathrm{m}$ thickness within $10 \mathrm{~min}$ of exposure time. On the other hand, during 5 and 30 min of exposure time, CoNi-layer thicknesses of 1.5 and $6 \mu \mathrm{m}$ were generated, respectively. The $M-H$ loops of the ascast single-phase $\mathrm{Fe}_{64} \mathrm{Co}_{8} \mathrm{~B}_{19.2} \mathrm{Si}_{4.8} \mathrm{Ta}_{4}$ amorphous wire and some wires after the growth of the CoNi hard phase with selected thicknesses are shown in figure 2. Note that the maximum magnetization value takes values between 0.91 and $0.43 \mathrm{~T}$ for the thickness interval of $0.0-6.0 \mu \mathrm{m}$ under the maximum applied field $H$ of $2 \mathrm{kA} / \mathrm{m}$. One should understand that the loop mainly denotes magnetic behaviour of the soft core once the magnetization of the harder shell is not achieved by the maximum applied field.

The low-field magnetization traces for biphase wires and the soft magnetic wire precursor are displayed in figure 2. It is assumed that $M-H$ curves mainly correspond to the soft phase (given that the $\mathrm{Fe}_{64} \mathrm{Co}_{8} \mathrm{~B}_{19.2} \mathrm{Si}_{4.8} \mathrm{Ta}_{4}$ amorphous core for which a coercive field, $H_{\mathrm{c}}$, of $40 \mathrm{~A} / \mathrm{m}$ is determined)

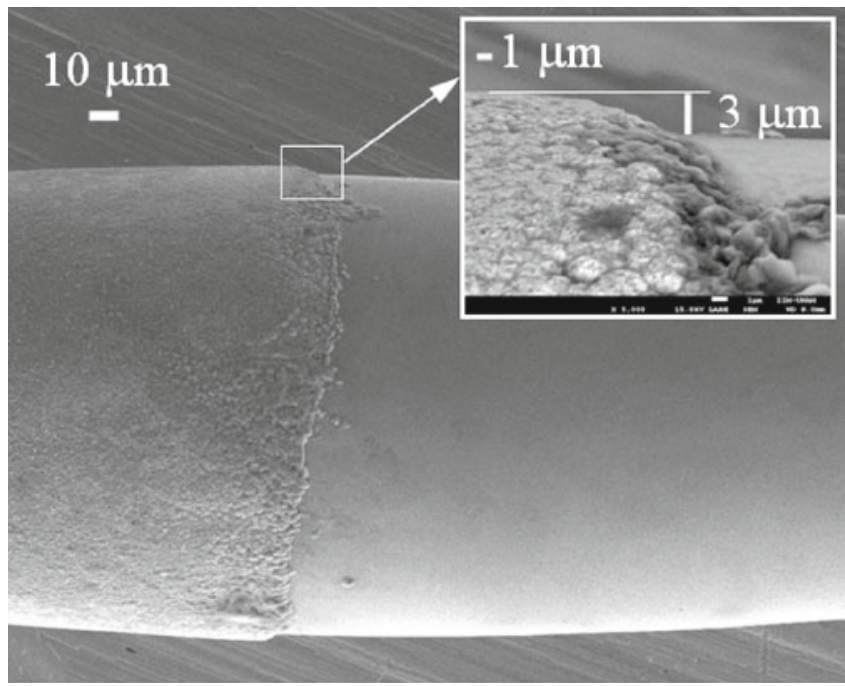

Figure 1. SEM images showing soft magnetic amorphous nucleus and hard magnetic CoNi layer within 10 min of exposure time. Inset shows CoNi layer thickness corresponding to $3 \mu \mathrm{m}$.
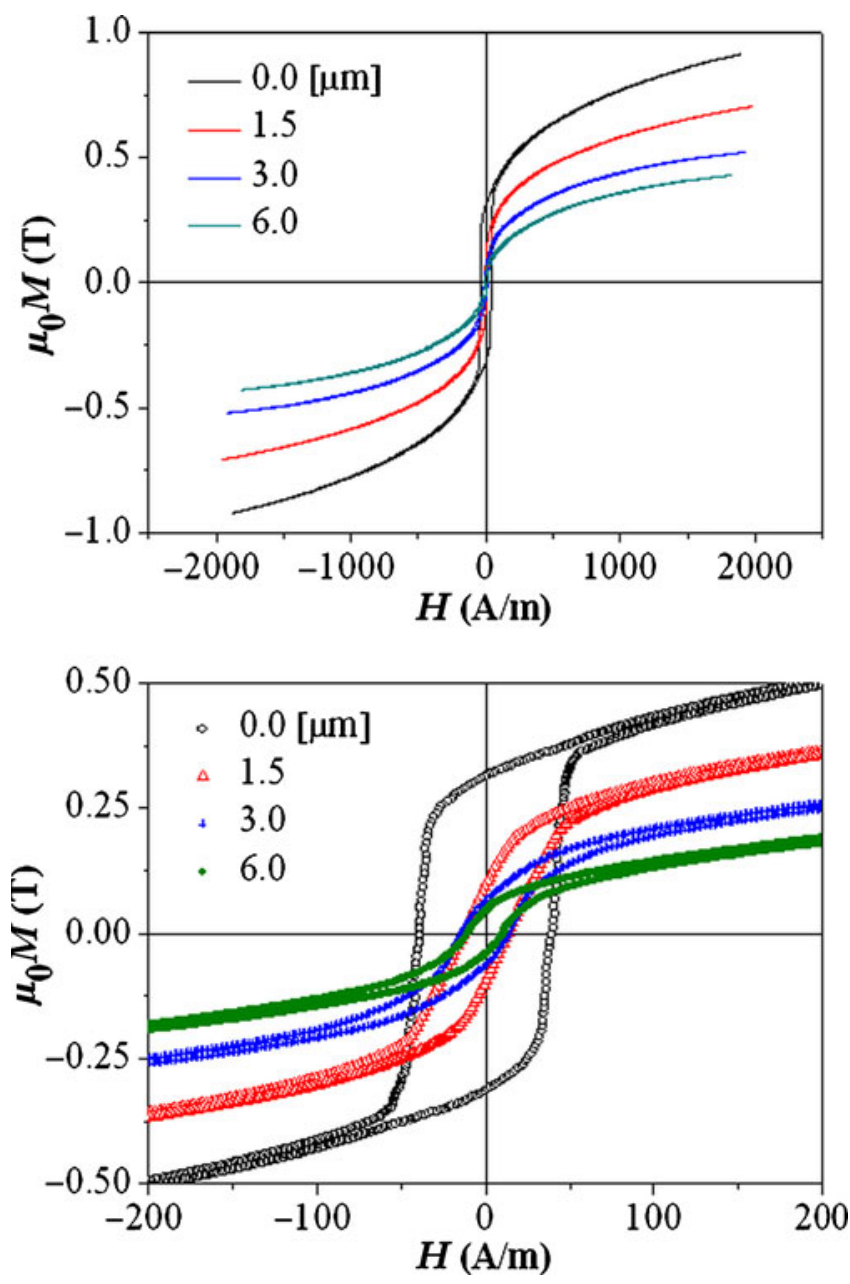

Figure 2. $M-H$ hysteresis loops for $\mathrm{Fe}_{64} \mathrm{Co}_{8} \mathrm{~B}_{19 \cdot 2} \mathrm{Si}_{4 \cdot 8} \mathrm{Ta}_{4}$ amorphous wire and same wire after growth of $\mathrm{Co}_{90} \mathrm{Ni}_{10}$ external layer with different thicknesses, $t$ (top), and a detail of loops to low-field (bottom). 
that, probably, the magnetization of the hard CoNi layer is not reached with the maximum field applied by device $(2 \cdot 1 \mathrm{kA} / \mathrm{m})$. It can be observed that in the lower portion of figure 2 since the thickness $t$ of the external crystalline phase increases, both remanence and coercivity decrease, resulting in a reduced hysteresis. It reflects an increasing rotational contribution to the wire's magnetization process, as a consequence of the development of out-of-axis magnetization in the wire central core. Anisotropy is seemingly induced by the presence of hard CoNi external layer and denotes the existence of a magnetic coupling between phases possibly due to magnetoelastic origin.

The spectroscopy behaviour for real and imaginary components of complex permeability for single and biphase amorphous wires is shown in figure $3(a, b)$ for variable thickness of the external CoNi layer. These curves show resolution of the active magnetization mechanisms through the frequency range (Valenzuela 2002). For instance, the $\mu_{\mathrm{re}}(f)$ plot for the as-cast amorphous wire $(t=0.0 \mu \mathrm{m}$, figure 3(a)) shows an initial plateau when frequency, $f$ values increase up to $10^{3} \mathrm{~Hz}$. The evolution of $\mu_{\text {re }}$ has been attributed to the reversible bulging of the magnetic domain walls (DWs) and thus, it can be associated with the initial permeability,

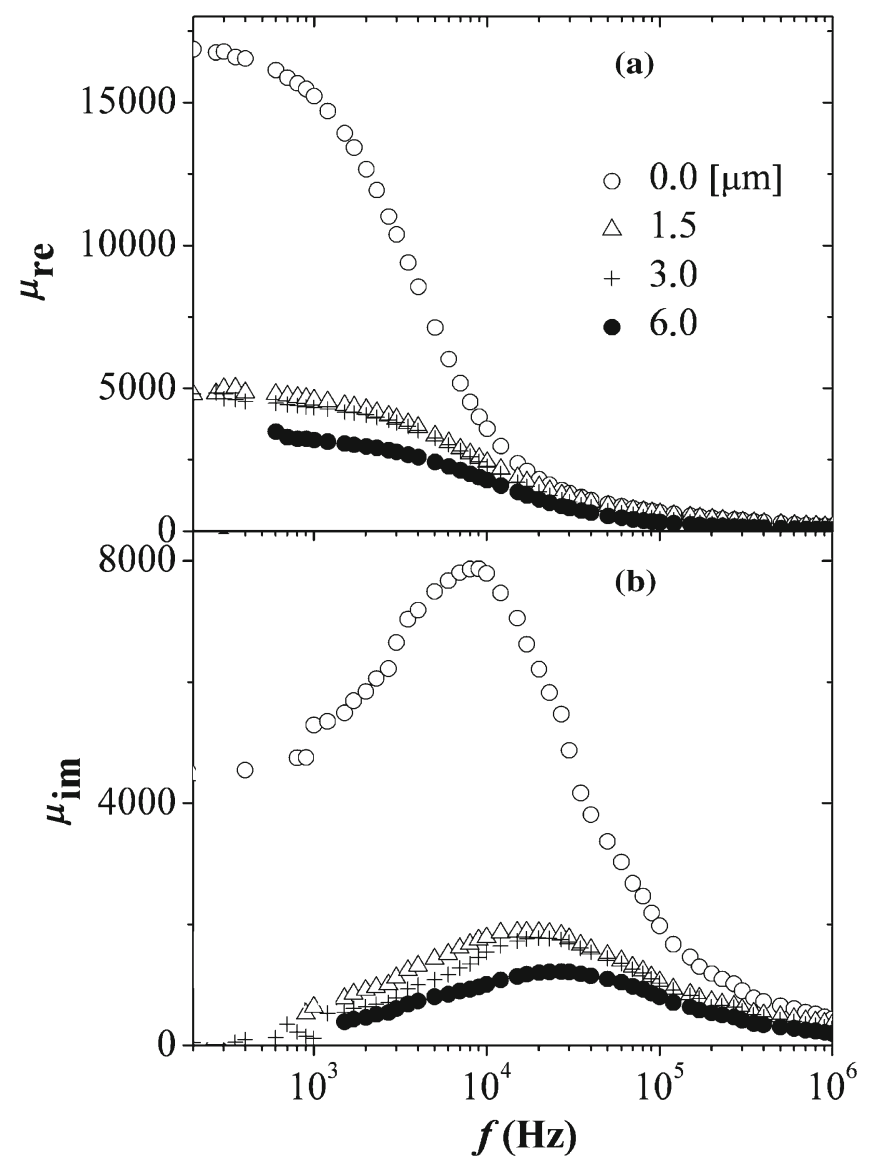

Figure 3. (a) Real and (b) imaginary permeability spectra for single and biphase wires with different hard layer thicknesses, $t$. $\mu_{\text {ini }}$ of the soft core (Chikazumi 1978). Further increase in $f$ causes a significant reduction in $\mu_{\mathrm{re}}(f)$, which can be attributed to a relaxation-type dispersion of the reversible bulging mechanism for which the DWs are no longer able to follow the a.c. magnetic variations. Beyond a threshold frequency, $f_{x}$ (or relaxation frequency), $\mu_{\text {re }}$ becomes very small, it reflects an increasing rotational contribution for the only active magnetization process for $f>f_{x}$ (Chikazumi 1978).

The CoNi layer thickness affects significantly the lowfield reversible magnetization process of soft core since $\mu_{\text {ini }}$ decreases from 16738 (as-cast amorphous wire, $t=0.0 \mu \mathrm{m}$ ) to $3205(t=6.0 \mu \mathrm{m})$. This marked reduction for $\mu_{\text {ini }}$ can be attributed to the increase in the out-of-axis anisotropy developed by the biphase wires with the increase in hard CoNi layer thickness as a consequence of the accumulation of mechanical stress appearing at the amorphous-wire/CoNilayer interface, for which, a higher CoNi layer thickness implies higher accumulated internal stresses.

On the other hand, the imaginary $\mu_{\text {im }}$ component (figure 3(b)) is associated with magnetic losses (hysteresis, eddy current or power losses) (Chikazumi 1978; Chen and Muñoz 1999). Consequently, the very presence of the external hard layer reduces significantly the losses (figure 2), confirming the low-frequency results in figure 3 . The maximum in $\mu_{\text {im }}$ is observed at frequency, $f_{x}$. For the present biphase wires, $f_{x}$ exhibits an increasing tendency (figure $3(\mathrm{~b})$ ) from $9 \mathrm{kHz}$ $(t=0.0 \mu \mathrm{m})$ to $27 \mathrm{kHz}(t=6.0 \mu \mathrm{m})$ and follows an inverse tendency with $t$, which confirms influence of the variable anisotropy on the active spin rotation mechanism for $f>f_{x}$, for which the product $\mu_{\mathrm{re}} f_{x}$ results in a constant value of $\sim 0.1 \mathrm{GHz}$. This converse correlation between $\mu_{\text {ini }}$ and $f_{x}$ is verified in figure 4 as a function of $t$. This indicates that a reduced $\mu_{\text {ini }}$ correlates to the rigidity of the magnetic domain wall, leading to higher $f_{x}$ values. A similar correlation between both parameters has also been found on ferrites as well as on amorphous ribbons and wires (Omata et al 1989; Nakamura 2000).

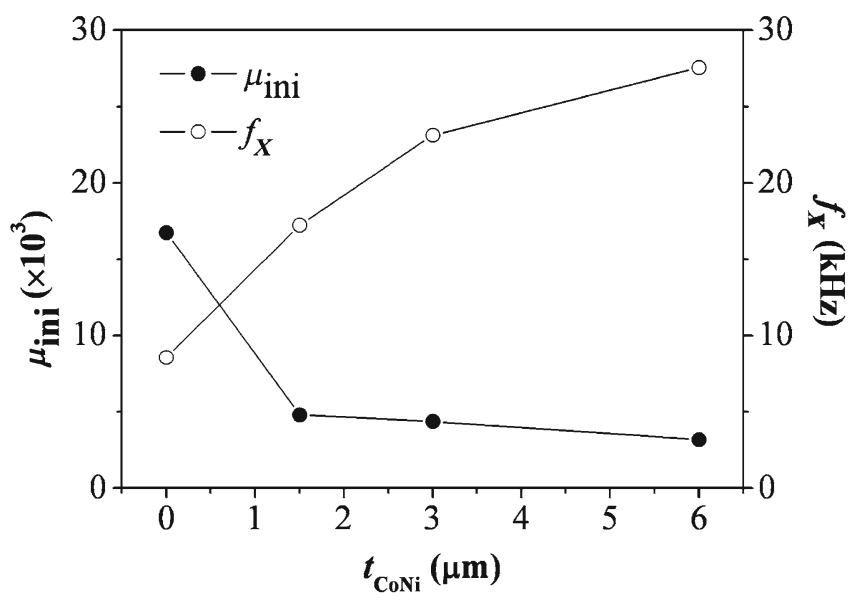

Figure 4. Plot of change in $\mu_{\text {ini }}$ and $f_{x}$ as a function of hard layer thickness, $t$. 


\section{Conclusions}

Biphase wire systems consisting of soft magnetic amorphous wire nucleus surrounded by a layer of hard magnetic $\mathrm{Co}_{90} \mathrm{Ni}_{10}$ of variable thickness were prepared by in-rotating water quenching method and subsequent electroplating technique. Low-field magnetization processes have been studied as a function of CoNi hard layer thickness. It is concluded that the external layer induces an out-of-plane magnetic anisotropy in the soft magnetic core that controls its magnetization process. Particularly, the losses are reduced, with a reduction in both coercivity and remanence, as well as a reduction in complex permeability.

\section{Acknowledgements}

One of the authors (SB) is grateful to Armando L Valencia and acknowledges the scholarship granted by DGEP-UNAM. (IB) acknowledges the financial support from Research Grant IN106808 PAPIIT-UNAM.

\section{References}

Butta M, Infante G, Ripka P, Badini-Confalonieri G and Vázquez M 2009 Rev. Sci. Instrum. 80083906

Chen D X and Muñoz J L 1999 IEEE Trans. Mag. 351906
Chikazumi S 1978 Physics of magnetism (New York: Krieger R E Pub. Co.)

Hernando A and Marín P 2005 Magnetic microwires: Manufacture, properties and applications, concise encyclopedia of magnetic and superconducting materials (ed.) $\mathrm{K} \mathrm{H} \mathrm{J}$ Buschow (Amsterdam: Elsevier) 2nd ed.

Konno Y, Kawashima K, Mohri K, Mizutani M, Kimma K, Ogasawara I and Takamido H 1990 IEEE Trans. J. Magn. Jpn. 5 1077

Nakamura T 2000 J. Appl. Phys. 88348

Omata Y, Sakakima H and Kugimiya K 1989 IEEE Trans. J. Magn. Jpn. 4518

Pirota K R, Provencio M, Garcia K L, Escobar-Galindo R, Mendoza Zelis P, Hernandez-Velez M and Vázquez M 2005 J. Magn. Magn. Mater. 290-291 68

Pirota K R, Hernandez-Velez M, Navas D, Zhukov A and Vázquez M 2004 Adv. Funct. Mater. 14266

Torrejón J $2008 \mathrm{PhD}$ dissertation, Autonomous University of Madrid, Madrid

Valenzuela R 2002 Mater. Res. Symp. 699127

Valenzuela R 2004 J. Alloys Compds 36940

Valenzuela R 2007 The frequency response of magnetic materials, in Magnetic materials: Current topics in amorphous wires, hard magnetic alloys, ceramics, characterization and modelling (ed.) I Betancourt (Kerala: Research SignPost)

Vázquez M 2007 Handbook of magnetism and advanced magnetic materials: Advanced magnetic microwires (eds) H Kronmüller and S Parking (Chichester: Wiley) Vol. 5, p. 2193

Vázquez M, Badini-Confalonieri G, Kraus L, Pirota K R and Torrejón J 2007 J. Non-Cryst. Solids 353763

Vázquez M, Castaño F, Ovari T A, Raposo V and Hernando A 2001 Sensors Actuators A: Physical 91112 